\title{
Further studies of QCD with sextet quarks
}

\author{
D. K. Sinclair ${ }^{* \dagger}$ \\ HEP Division, Argonne National Laboratory, 9700 South Cass Avenue, Argonne, Illinois 60439, \\ USA \\ E-mail: dks@hep.anl.gov
}

\section{J. B. Kogut}

Department of Energy, Division of High Energy Physics, Washington, DC 20585, USA

and

Department of Physics - TQHN, University of Maryland, 82 Regents Drive, College Park, MD 20742, USA

E-mail: jbkoguteumd.edu

\begin{abstract}
We continue our simulations of QCD with 2 flavours of colour-sextet quarks as a model for walking technicolor. QCD with 3 flavours of colour-sextet quarks is also studied for comparison with the 2-flavour theory. We simulate these theories at finite temperatures $\mathrm{T}$, using lattices with a finite extent $N_{t} a=1 / T$ in the (Euclidean) time direction. The lattice coupling at the chiral-symmetryrestoration transition is measured as a function of $N_{t}$. If this is indeed a finite-temperature transition, the evolution of this coupling with $N_{t}$ as $N_{t} \rightarrow \infty$ and hence the lattice spacing $a \rightarrow 0$ should be described by asymptotic freedom. If so, the theory is QCD-like and walking. If, however, this coupling approaches a constant non-zero value in the large $N_{t}$ limit, the transition is a bulk transition and the continuum theory is conformal. For the 2-flavour theory, the coupling does show a significant decrease between $N_{t}=8$ and $N_{t}=12$, favouring the walking scenario. However, preliminary results are that the change is less than that predicted by asymptotic freedom. For the 3-flavour case, which is expected to be conformal, there is still a significant decrease in the coupling between $N_{t}=6$ and $N_{t}=8$, indicating that we are not yet at large enough $N_{t}$.
\end{abstract}

31st International Symposium on Lattice Field Theory - LATTICE 2013

July 29 - August 3, 2013

Mainz, Germany

\footnotetext{
* Speaker.

$\dagger$ This research was supported in part by US Department of Energy contracts DE-AC02-06CH11357 and DE-FG02-12ER41871
} 


\section{Introduction}

In the standard Standard Model, the Higgs field is an elementary complex scalar, whose couplings to the $S U(2) \times U(1)$ electroweak gauge bosons $\gamma, W^{ \pm}, Z$ are prescribed by gauge invariance. It has Yukawa couplings to the quarks and leptons, and a quartic self-coupling. Its quadratic selfcoupling with dimensions of mass-squared is negative, so that it develops a vacuum expectation $v$ which breaks $S U(2) \times U(1)$ spontaneously. The $W^{ \pm}$and $Z$ gain masses by 'eating' the 3 Goldstone bosons. $v$ gives masses to the fermions through the Yukawa couplings. The remaining (radial) component of the Higgs field is the so-called Higgs particle. The recent discovery of a light Higgs candidate at the LHC (mass $\approx 125 \mathrm{GeV}$ ) makes attempts to understand the Higgs sector of the Standard Model timely.

We consider the possibility that this simplest model of the Higgs sector of the Standard Model is merely an effective field theory, and that the Higgs fields are composite. The simplest theories of this type are Technicolor theories [1] 2]. These are QCD-like gauge theories with massless (techni-)quarks, where the (techni-)pions play the role of the Higgs field giving masses to the $W \mathrm{~s}$ and $Z$. Technicolor theories which are merely scaled-up QCD are not phenomenologically viable. It can be argued that Walking Technicolor theories [3, 4, 5, 6], where the gauge group and fermion content are such that there is a range of length/mass scales where the running coupling evolves very slowly, might overcome these problems [ [ , 8, , 2, 10]. We are trying to identify gauge theories that walk. If we find such a theory, we then need to check if it is indeed phenomenologically viable. An important aspect of an acceptable theory is that it must describe the light $\left(m_{H} \approx \frac{1}{2} v\right)$ Higgs-like particle observed at the LHC. Gauge theories with fermion content such that the theory is asymptotically free, but the 1- and 2-loop contributions to the $\beta$-function have opposite signs, are expected to be either conformal or walking.

Our candidate theory is (techni-)QCD with 2 massless (techni-)colour-sextet (techni-)quarks, which could be either walking or conformal. We contrast this theory with the 3-(techni-)flavour version, which is expected to be conformal. QCD with 2 colour-sextet quarks has 3 Goldstone bosons - the correct number to give mass to the $W \mathrm{~s}$ and $Z$ with none left over. In this sense it is minimal. For other arguments as to why this theory might be of interest see [11, 12].

Because chiral symmetry breaking and confinement occur at very different scales in this theory, one expects (techni-)hadrons associated with both scales. Hence light hadrons at the confinement scale could well have masses $<f_{\pi(T C)}=v$. Thus it could potentially have light Higgs-like particles other than the dilaton.

Other groups working on this model include DeGrand et al. [13, 14, 15, 16, 17, 18, 19] and Fodor et al. [20, 21, 22, 23, 24, 25, 26].

We simulate lattice QCD with 2 sextet quarks at finite temperature, and measure the running of the couplings at the deconfinement and chiral transitions, as the lattice spacing is varied [27, 28]. On lattices with finite temporal extent $N_{t} a$ and spatial extent $N_{s} a$ with $N_{s}>>N_{t}$, the temperature is $T=1 / N_{t} a$. If our transitions are finite temperature transitions, they will remain at fixed temperatures as the lattice spacing $a$ is varied. If we increase $N_{t} \rightarrow \infty$ at either transition, $a \rightarrow 0$. The bare (lattice) coupling $g$ at the transition should approach zero as $N_{t} \rightarrow \infty$ in the manner described by the (perturbative) $\beta$-function. If on the other hand, the transition is a bulk transition, $g$ will approach a non-zero limit as $N_{t} \rightarrow \infty$. In this case the field theory is conformal. Our simulations use the Wil- 
son (plaquette) gauge action and the unimproved staggered fermion action. The RHMC simulation algorithm is used to tune to a number $N_{f}$ of fermions which is not a multiple of $4\left(N_{f}=2\right)$.

Since the deconfinement transition occurs at a value of $\beta=6 / g^{2}$ that is too small to observe asymptotic freedom, for the $N_{t}$ s we use, we concentrate our effort on the chiral-transition- $\beta, \beta_{\chi}$.

We simulate the $N_{f}=2$ theory on lattices with $N_{t}=4,6,8,12$ and hope to extend this to larger $N_{t}$. Preliminary results indicate that $\beta_{\chi}\left(N_{t}=12\right)$ is significantly larger than $\beta_{\chi}\left(N_{t}=8\right)$, but by less than what the 2-loop $\beta$-function would predict.

We simulate the $N_{f}=3$ theory on lattices with $N_{t}=4,6,8$ and will extend this to $N_{t}=12$ [29, 28]. Preliminary results indicate that $\beta_{\chi}\left(N_{t}=8\right)$ is significantly greater than $\beta_{\chi}\left(N_{t}=6\right)$ which would indicate that we are not yet at large enough $N_{t}$, since we expect $\beta_{\chi}$ to approach a non-zero constant at large $N_{t}$. Even if this theory is QCD-like, asymptotic freedom would predict that the change in $\beta_{\chi}$ between $N_{t}=6$ and $N_{t}=8$ should be too small to be measured in our current simulations. Again we simulate using the RHMC algorithm since $N_{f}=3$ is not a multiple of 4 .

\section{Lattice QCD with 2 flavours of colour-sextet quarks}

\subsection{Simulations with $N_{t}=8$}

Our $N_{t}=8$ simulations were performed primarily on a $16^{3} \times 8$ lattice. Simulations performed on a $24^{3} \times 8$ lattice at $\beta=6.7$ and $\beta=6.9$, both with $m=0.0025$ (the smallest mass we use, indicate that finite lattice size errors are small on lattices of spatial extent $N_{s}=16$. We have no new $16^{3} \times 8$ results since Lattice 2012 [28].

Our $16^{3} \times 8$ simulations were performed at $m=0.02, m=0.01, m=0.005$, and $m=0.0025$ to allow performance of chiral extrapolations. In the neighbourhood of the chiral transition, $6.6 \leq$ $\beta \leq 6.8$, we performed runs of 50,000 length- 1 trajectories at $m=0.02, m=0.01$, and $m=0.005$ for each $\beta$ and $m$ for $\beta$ s spaced at 0.02 intervals. At $m=0.0025$ we have performed runs of 100,000 trajectories for each $\beta$ in this range.

Since it is difficult if not impossible to extrapolate either the unsubtracted or subtracted chiral condensates to zero quark mass with sufficient reliability to determine the position of the chiralsymmetry-restoration phase transition accurately, we estimate the value of $\beta_{\chi}$ from the peaks in the (disconnected) chiral susceptibility:

$$
\chi_{\bar{\psi} \psi}=\frac{V}{T}\left[\left\langle(\bar{\psi} \psi)^{2}\right\rangle-\langle\bar{\psi} \psi\rangle^{2}\right]
$$

extrapolated to $m=0$. Since the positions of the peaks in these susceptibilities show little mass dependence, we take the position of the peak for $m=0.0025$ as our estimate of $\beta_{\chi}$. This gives $\beta_{\chi}=6.69(1)$. Ferrenberg-Swendsen reweighting was used to interpolate between $\beta$ values to determine this peak. We refer to our Lattice 2012 talk [28] for graphs. For $m=0.0025$ and $\beta=6.7$, and for $\beta=6.9$, these susceptibilities are consistent with those measured on a $24^{3} \times 8$ lattice.

\subsection{Simulations with $N_{t}=12$}

We are now extending our simulations at $N_{t}=12$ on $24^{3} \times 12$ lattices, with quark masses $m=$ $0.01, m=0.005$, and $m=0.0025$. In the neighbourhood of the chiral transition $6.6 \leq \beta \leq 6.9$ we 
perform simulations at $\beta$ s spaced by $\delta \beta=0.02$. So far we have simulated 20,000 - 100,000 length1 trajectories at each $(\beta, m)$ in this range. Figure 1 shows the unsubtracted chiral condensates $\langle\bar{\psi} \psi\rangle$ measured in these simulations. Note that, although these suggest that the condensate will vanish in the chiral limit for $\beta$ sufficiently large, any attempt to extrapolate to $m=0$ to estimate $\beta_{\chi}$ would be plagued with systematic uncertainties. Using subtracted condensates improves the situation, but not sufficiently to extract $\beta_{\chi}$ with the needed precision.

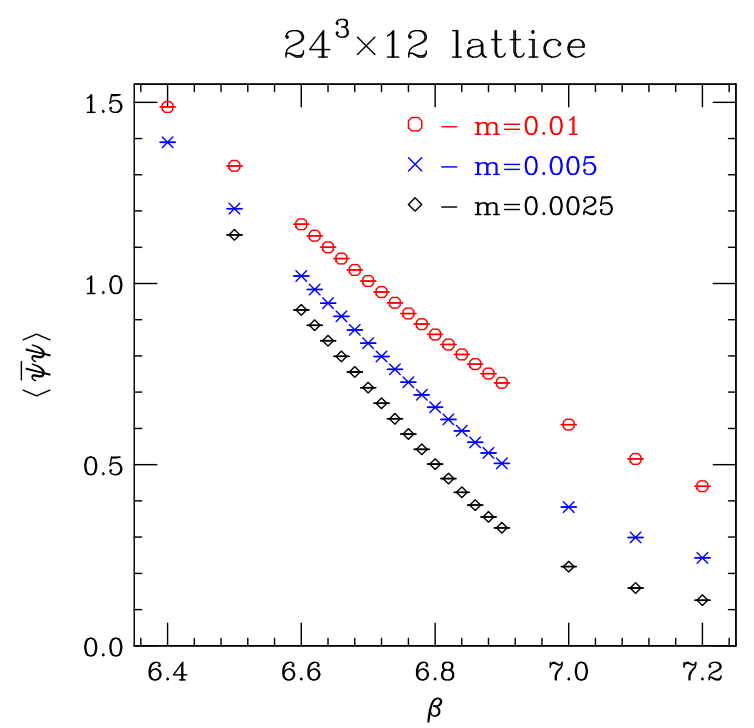

Figure 1: Chiral condensates on a $24^{3} \times 12$ lattice.

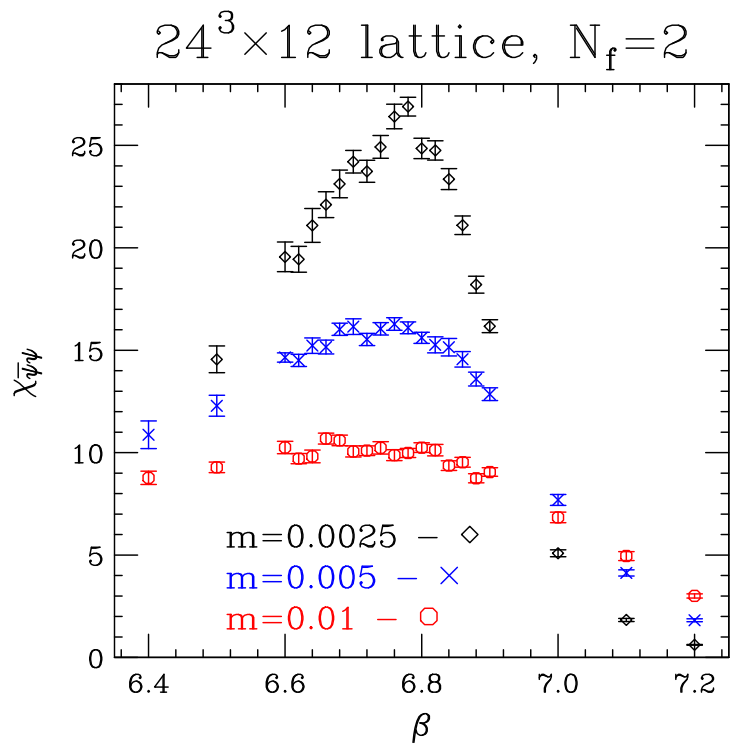

Figure 2: Chiral susceptibilities on a $24^{3} \times 12$ lattice.

To determine the position $\beta_{\chi}$ of the chiral transition with sufficient accuracy, we examine the peak in the (disconnected) chiral susceptibility as a function of mass. These susceptibilities are shown in figure 2. Both the $m=0.005$ and $m=0.0025$ susceptibilities show peaks. While the 'data' is consistent with there being little mass dependence of the position of the peaks, it is not yet compelling. More statistics is needed. Our best estimate of $\beta_{\chi}$ from the current data is $\beta_{\chi}=6.78(2)$. This implies that:

$$
\beta_{\chi}\left(N_{t}=12\right)-\beta_{\chi}\left(N_{t}=8\right)=0.09(2)
$$

compared with the 2-loop perturbative prediction

$$
\beta_{\chi}\left(N_{t}=12\right)-\beta_{\chi}\left(N_{t}=8\right) \approx 0.12
$$

\subsection{Simulations on $24^{3} \times N_{t}$ lattices}

In addition to our fixed $N_{t}$ simulations, we simulate this 2-flavour theory on $24^{3} \times N_{t}$ lattices with $N_{t} \leq 24$ at fixed $\beta$, to search for evidence of a transition back to the chirally broken phase, as $N_{t}$ is increased. We choose $\beta=6.9$. This beta was chosen since, if the evolution of $\beta_{\chi}$ is governed by the 2-loop $\beta$-function, $\beta_{\chi} \approx 6.9$ for $N_{t}=18$. Hence we should see evidence for the chiral transition as $N_{t}$ is increased. 
At present, we run on $24^{3} \times 8,24^{3} \times 10,24^{3} \times 12,24^{3} \times 18,24^{3} \times 20,24^{3} \times 22$ and $24^{4}$ lattices at $m=0.005, m=0.0025$ and $m=0.00125$. We plan runs on $24^{3} \times 14$ and $24^{3} \times 16$ lattices. Figure 3 shows the unsubtracted and subtracted chiral condensates as functions of $N_{t}$ for these runs. We follow Fodor et al., defining a subtracted chiral condensate by:

$$
\langle\bar{\psi} \psi\rangle_{\text {sub }}=\langle\bar{\psi} \psi\rangle-\left(m_{V} \frac{\partial}{\partial m_{V}}\langle\bar{\psi} \psi\rangle\right)_{m_{V}=m}
$$

where $m_{V}$ is the valence quark mass [21]. We should try other schemes, since this data is inconclusive. We have also looked at the chiral susceptibilities, but with our present statistics we have not seen a peak.

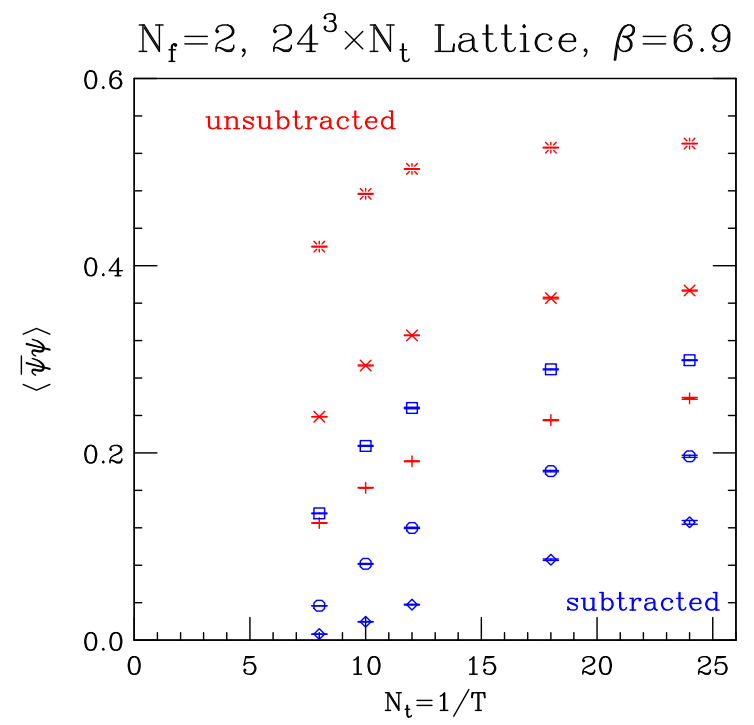

Figure 3: Chiral condensates on $24^{3} \times N_{t}$ lattices at $\beta=6.9$. From top to bottom, the masses are $m=$ Figure 4: Chiral susceptibilities for $N_{f}=3$ on a $0.005, m=0.0025$ and $m=0.00125$.

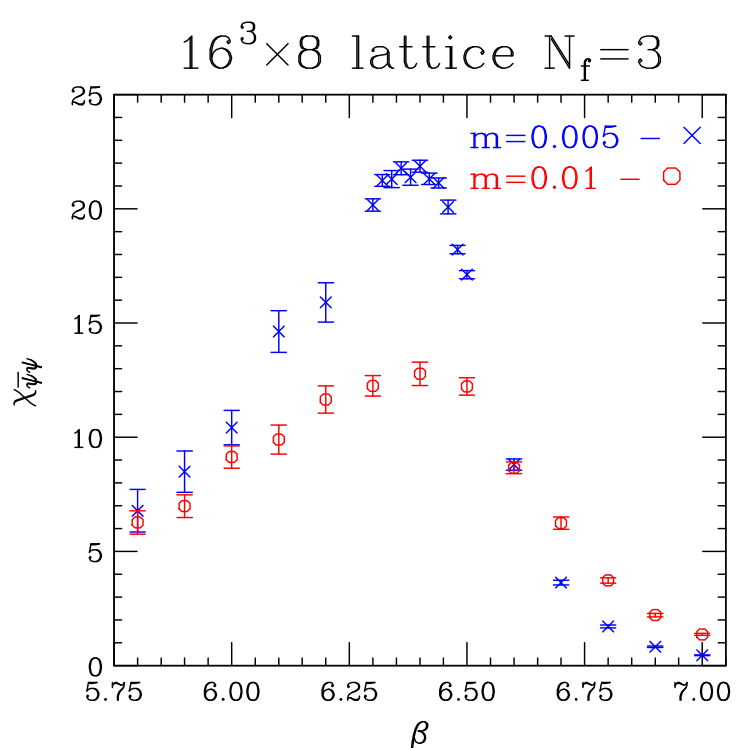

Figure 4: Chira
$16^{3} \times 8$ lattice.

\section{Lattice QCD with 3 flavours of colour-sextet quarks}

We simulate lattice QCD with 3 light quark flavours on $16^{3} \times 8$ lattices. Our simulations are performed at $m=0.01$ and $m=0.005$. For $6.3 \leq \beta \leq 6.5$, which is close to the chiral transition, and $m=0.005$, we have performed runs of 100,000 trajectories at intervals of 0.02 in $\beta$ at each $\beta$ in this range. Figure $\bigoplus$ shows the chiral susceptibilities for these runs. From this we estimate that $\beta_{\chi}=6.38(2)$, somewhat larger than that for $N_{t}=6$.

\section{Discussions and Conclusions}

We are simulating lattice QCD with 2 light colour-sextet quarks at finite temperature, to test whether it has an infrared fixed-point and is thus conformal, or if it is QCD-like, but with a slowlyevolving running coupling constant, i.e. if it 'walks' and is thus a Walking-Technicolor candidate. 


\begin{tabular}{|c|c|c|}
\hline$N_{t}$ & \multicolumn{1}{|c|}{$\beta_{d}$} & $\beta_{\chi}$ \\
\hline 4 & $5.40(1)$ & $6.3(1)$ \\
6 & $5.54(1)$ & $6.60(2)$ \\
8 & $5.65(1)$ & $6.69(1)$ \\
12 & $5.8(1)$ & $6.78(2)$ \\
\hline
\end{tabular}

Table 1: $N_{f}=2$ deconfinement and chiral transitions for $N_{t}=4,6,8,12$.

We have extended our simulations at $N_{t}=12$. As seen in table 11 above, the chiral phase transition moves to larger $\beta$ as $N_{t}$ is increased. However, $\beta_{\chi}\left(N_{t}=12\right)-\beta_{\chi}\left(N_{t}=8\right)=0.09(2)$ compared with $\approx 0.12$ predicted using the 2 -loop perturbative $\beta$-function. More statistics is needed to ratify this result. We will also test if this is a finite size effect. It is possible that the 3-loop term in the $\beta$ function is large in this lattice regularization. Larger $N_{t}$ s might be needed to clarify this issue. Note that the chiral susceptibility is very sensitive to long 'time'-constant modes describing the system's evolution. These often have small amplitudes, so that they are not evident in other observables.

Our simulations of the 3-flavour theory at $N_{t}=8$ indicate that the increase in $\beta, \beta_{\chi}\left(N_{t}=\right.$ $8)-\beta_{\chi}\left(N_{t}=6\right)$ is appreciably larger than the $\approx 0.0025$ predicted by 2 -loop perturbation theory. Nor can we see any evidence that $\beta_{\chi}\left(N_{t}\right)$ is approaching a non-zero constant as $N_{t} \rightarrow \infty$ as expected, since the this theory is expected to be conformal. We will therefore need to perform simulations at larger $N_{t} . N_{t}=12$ simulations are planned for 2014. We will also extend our $N_{t}=6$ simulations to obtain a better estimate of the position of its chiral phase transition.

To study the spectrum of the 2-flavour theory at zero temperature, we need to restrict ourselves to the region $\beta<\beta_{d}$, the $\beta$ at the deconfinement transition. For this $\beta$ to also lie in the weakcoupling domain, so that we can make contact with the continuum will require rather large lattices. Since our experience with the chiral transition suggests that the crossover from strong- to weakcoupling occurs somewhere in the regime $\beta=6.3-6.6$ while $\beta_{d}\left(N_{t}=12\right) \approx 5.75$, we estimate that we will require lattices whose smallest dimension $N_{s}=48$ or larger. For zero temperature physics, $f_{\pi(T C)}$ is the relevant scale. We know that $f_{\pi(T C)}=v \approx 246 \mathrm{GeV}$, so $m_{\mathrm{Higgs}} \approx \frac{1}{2} f_{\pi(T C)}$. This puts a severe constraint on any model of Higgs dynamics, something that we will test in our model.

We also plan to simulate other candidate Walking Technicolor models. For example, we are planning to study is $S U(2)$ gauge theory with 3 adjoint Majorana/Weyl quarks, starting in 2014. A good summary of the current state of lattice simulations of various candidate theories is contained in the review talk by Julius Kuti at Lattice 2013 [30].

\section{Acknowledgements}

These simulations are performed on Hopper, Carver and Edison at NERSC, Kraken at NICS, and Fusion and Blues at LCRC, Argonne. We thank Julius Kuti for helpful insights, in particular his suggestion that we perform simulations at a fixed $\beta$ and $N_{s}$, for a range of $N_{t}$ values.

\section{References}

[1] S. Weinberg, Phys. Rev. D 19, 1277 (1979). 
[2] L. Susskind, Phys. Rev. D 20, 2619 (1979).

[3] B. Holdom, Phys. Rev. D 24, 1441 (1981).

[4] K. Yamawaki, M. Bando and K. i. Matumoto, Phys. Rev. Lett. 56, 1335 (1986).

[5] T. Akiba and T. Yanagida, Phys. Lett. B 169, 432 (1986).

[6] T. W. Appelquist, D. Karabali and L. C. R. Wijewardhana, Phys. Rev. Lett. 57, 957 (1986).

[7] T. Appelquist and F. Sannino, Phys. Rev. D 59, 067702 (1999) [hep-ph/9806409].

[8] S. D. H. Hsu, F. Sannino and J. Schechter, Phys. Lett. B 427, 300 (1998) [hep-th/9801097].

[9] M. Kurachi and R. Shrock, Phys. Rev. D 74, 056003 (2006) [hep-ph/0607231].

[10] T. Appelquist et al. [LSD Collaboration], Phys. Rev. Lett. 106, 231601 (2011) [arXiv:1009.5967 [hep-ph]].

[11] F. Sannino, K. Tuominen, Phys. Rev. D71, 051901 (2005). [hep-ph/0405209].

[12] D. D. Dietrich, F. Sannino, K. Tuominen, Phys. Rev. D72, 055001 (2005). [hep-ph/0505059].

[13] Y. Shamir, B. Svetitsky and T. DeGrand, sextet fermions," Phys. Rev. D 78, 031502 (2008) [arXiv:0803.1707 [hep-lat]].

[14] T. DeGrand, Y. Shamir and B. Svetitsky, Phys. Rev. D 79, 034501 (2009) [arXiv:0812.1427 [hep-lat]].

[15] T. DeGrand, Phys. Rev. D 80, 114507 (2009) [arXiv:0910.3072 [hep-lat]].

[16] T. DeGrand, Y. Shamir and B. Svetitsky, Phys. Rev. D 82, 054503 (2010) [arXiv:1006.0707 [hep-lat]].

[17] T. DeGrand, Y. Shamir and B. Svetitsky, Phys. Rev. D 87, 074507 (2013) [arXiv:1201.0935 [hep-lat]].

[18] T. DeGrand, Y. Shamir and B. Svetitsky, Phys. Rev. D 88, 054505 (2013) [arXiv:1307.2425 [hep-lat]].

[19] Talk presented by Y. Shamir at Lattice 2013 (2013).

[20] Z. Fodor, K. Holland, J. Kuti, D. Nogradi and C. Schroeder, JHEP 0911, 103 (2009) [arXiv:0908.2466 [hep-lat]].

[21] Z. Fodor, K. Holland, J. Kuti, D. Nogradi and C. Schroeder, arXiv:1103.5998 [hep-lat].

[22] Z. Fodor, K. Holland, J. Kuti, D. Nogradi, C. Schroeder and C. H. Wong, Phys. Lett. B 718, 657 (2012) [arXiv:1209.0391 [hep-lat]].

[23] Z. Fodor, K. Holland, J. Kuti, D. Nogradi, C. Schroeder and C. H. Wong, PoS LATTICE 2012, 024 (2012) [arXiv:1211.6164 [hep-lat]].

[24] Z. Fodor, K. Holland, J. Kuti, D. Nogradi, C. Schroeder and C. H. Wong, PoS LATTICE 2012, 025 (2012) [arXiv:1211.3548 [hep-lat]].

[25] Talk presented by K. Holland at Lattice 2013 (2013).

[26] Talk presented by C. H. Wong at Lattice 2013 (2013).

[27] J. B. Kogut and D. K. Sinclair, Phys. Rev. D 84, 074504 (2011) [arXiv:1105.3749 [hep-lat]].

[28] D. K. Sinclair and J. B. Kogut, PoS LATTICE 2012, 026 (2012) [arXiv:1211.0712 [hep-lat]].

[29] J. B. Kogut and D. K. Sinclair, Phys. Rev. D 85, 054505 (2012) [arXiv:1111.3353 [hep-lat]].

[30] J. Kuti, plenary talk at Lattice 2013 (2013). 